A machine learning algorithm predicts molecular subtypes in pancreatic ductal adenocarcinoma with differential response to gemcitabine-based versus FOLFIRINOX chemotherapy

Georgios Kaissis $^{1 \pi}$, Sebastian Ziegelmayer ${ }^{1 \pi}$, Fabian Lohöfer ${ }^{1 \pi}$, Katja Steiger ${ }^{2}$, Hana Algül ${ }^{3}$, Alexander Muckenhuber ${ }^{2}$, Hsi-Yu Yen², Ernst Rummeny ${ }^{1}$, Wilko Weichert ${ }^{2}$, Roland Schmid ${ }^{3}$, Helmut Friess ${ }^{4}$, Jens T. Siveke ${ }^{5,6}$ and Rickmer Braren ${ }^{1 *}$

${ }^{1}$ Department of diagnostic and interventional radiology, School of Medicine, Technical University of Munich, Munich, Germany;

${ }^{2}$ Department of Pathology, School of Medicine, Technical University of Munich, Munich, Germany;

${ }^{3}$ Department of Internal Medicine II, School of Medicine, Technical University of Munich, Munich, Germany;

${ }^{4}$ Department of Surgery, School of Medicine, Technical University of Munich, Munich, Germany;

${ }^{5}$ Division of Solid Tumor Translational Oncology, West German Cancer Center, University Hospital Essen, Essen, Germany.

${ }^{6}$ German Cancer Consortium (DKTK) and German Cancer Research Center (DKFZ), Heidelberg, Germany 


\section{Feature extraction}

Tumors were manually segmented by consensus reading as detailed in the main manuscript. Radiomic features were extracted from the quality-controlled 3-dimensional volumes as follows: Intensity discretization was performed to a fixed bin number of 32 bins for all scans as described in (1). No normalization was performed. Value plausibility was ascertained for all parameters. Images were spatially resampled to $3 \times 3 \times 3 \mathrm{~mm}$ using the BSpline interpolator. No resegmentation was performed. Radiomic features were derived using PyRadiomics v. 2.1.1 (2) yielding 19 first order statistics, 16 3D shape-based, 10 2D shape based, 24 Gray Level Cooccurence Matrix, 16 Gray Level Run Length Matrix, 16 Gray Level Size Zone Matrix, 5 Neighbouring Gray Tone Difference Matrix and 14 Gray Level Dependence Matrix features as well as Laplacian of Gaussian-filtered, wavelet-decomposition-based (using the coiflet 1 function), square, exponential, gradient, square-root, logarithm and local binary-pattern filtered versions of these features. Laplacian of Gaussian-filtering was performed with a kernel (sigma) of $3 \mathrm{~mm}$. GLCM and GLRLM were extracted using the default settings (separately for each direction then averaged). Feature descriptions can be found in the PyRadiomics documentation (3). Feature extraction and processing was carried out according to the IBSI manual v. 7 (4). 1606 features were extracted in total.

\section{Feature preprocessing}

The following radiomic features were excluded: Features yielding nil, constant or-missing values. Furthermore, tumors were segmented a second time after 2 weeks by the same observers to test for repeated segmentation stability. The intra-class-correlation coefficient (two-way mixed effects model/ consistency as described by McGraw and Wong (5)) was calculated and features yielding inter-segmentation values below 0.8 were excluded. In total, the following 40 reproducible and stable features remained: 


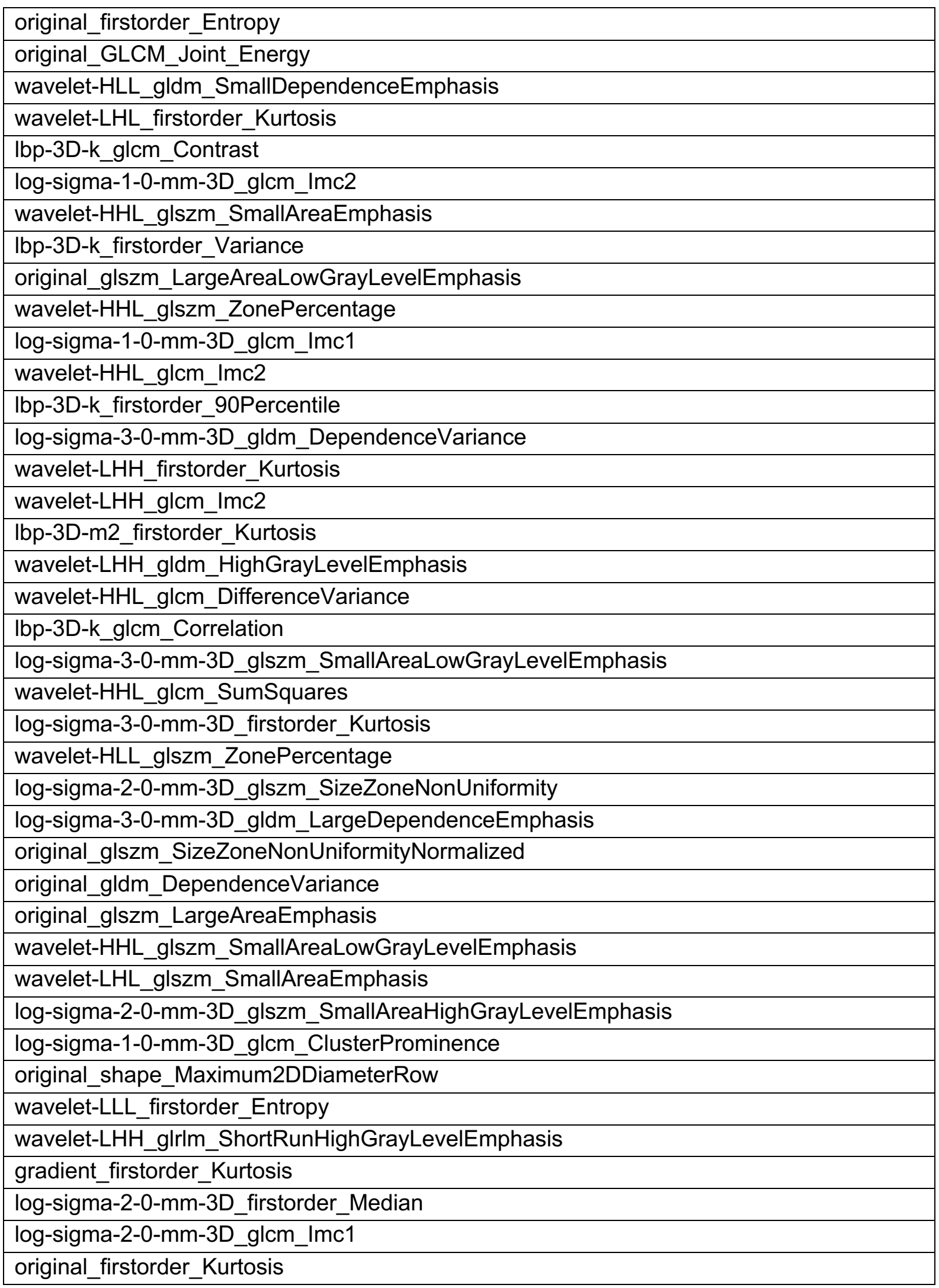

Potential intercorrelation between the relevant features selected by the algorithm was explored

by calculating Spearman's rho between their values. The correlation between the features was

found to be weak (as defined by Dancey and Reidy (6)) at best, signifying feature independence. A heatmap of feature correlation is seen below: 


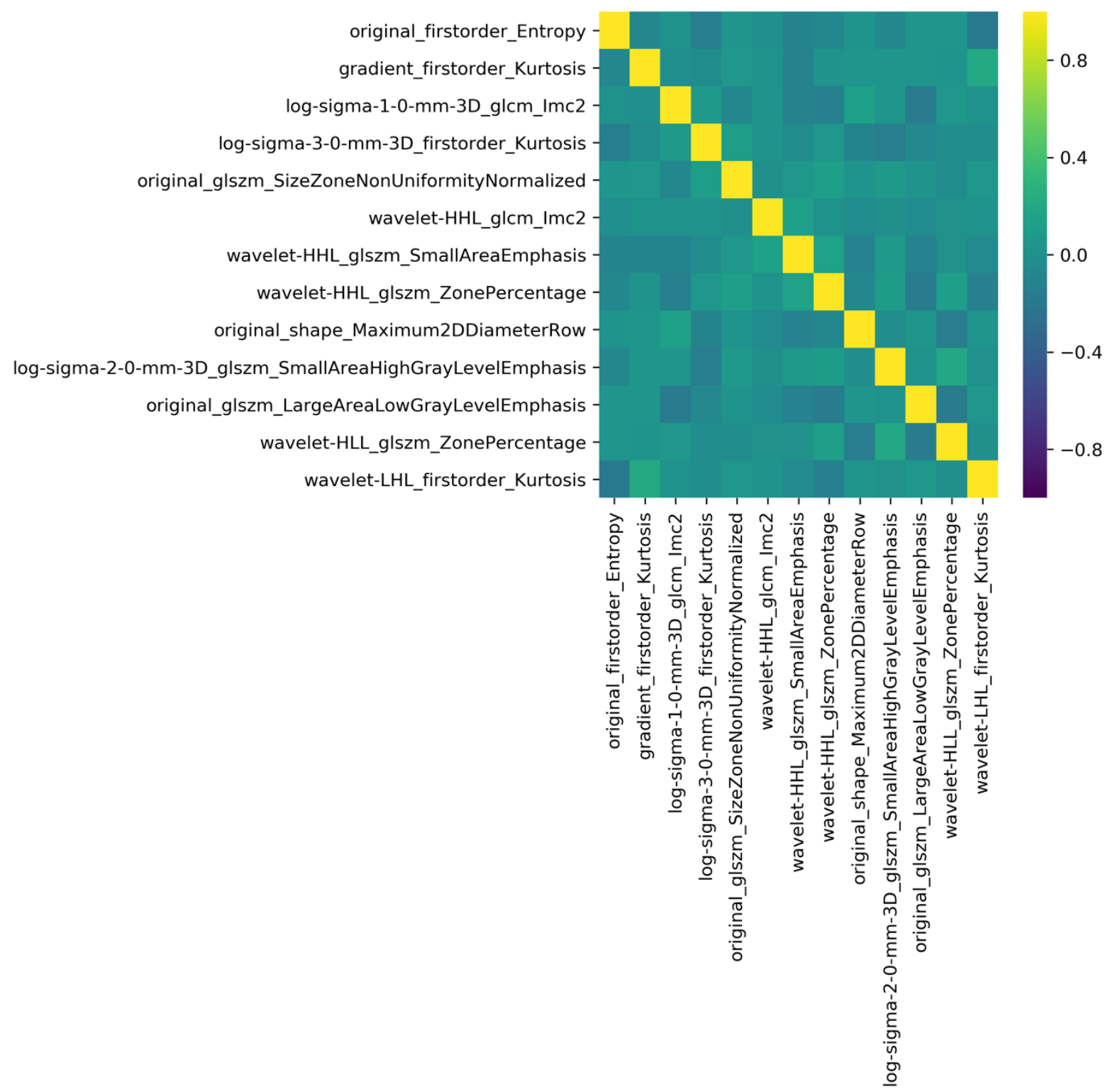

\section{Feature engineering and machine learning modeling}

Machine learning modeling was performed using the Python programming language version

3.7. The following packages were used: Pandas 0.23.4, Numpy 1.16.2, Matplotlib 3.0.3, Seaborn 0.9.0, Scikit Learn 0.20.3, Lifelines 0.21.0, Statsmodels 0.9.0, XGBoost 0.90., ScikitSurvival 0.9.

Feature values were normalized to a range of $(0,1)$ using the StandardScaler package implemented in sklearn.preprocessing.

Because of the small dataset size, the use of automatic hyperparameter tuning and nested cross-validation was eschewed. Some manual hyperparameter modifications were made to reduce model variance and increase overfitting resistance: tree complexity penalization was 
applied to the leaf values in the form of $L 2$ regularization and shrinkage regularization was introduced by reducing the learning rate.

For training and testing, the estimator was fit and tested using stratified shuffle/split crossvalidation with 10 splits of $70 \% / 30 \%$ (train/test) of the dataset. For testing survival prediction, a manual train/test set was built using interleaving to preserve the time-series structure and a stochastic Gradient Boosted Regression Tree algorithm was used. The learning rate was set at 0.01 and individual trees were built using $90 \%$ of the rows. Cox proportional hazards was used as a loss function to optimize and trees were split based on the Friedman MSE criterion. The concordance index between the predictions of the model on the testing set and the actual survival times was calculated.

For all other survival analyses, the stratified shuffle/split cross-validation technique was used. The survival curves stratified by radiomic signature only and by both, radiomic signature and chemotherapy can be found below: 


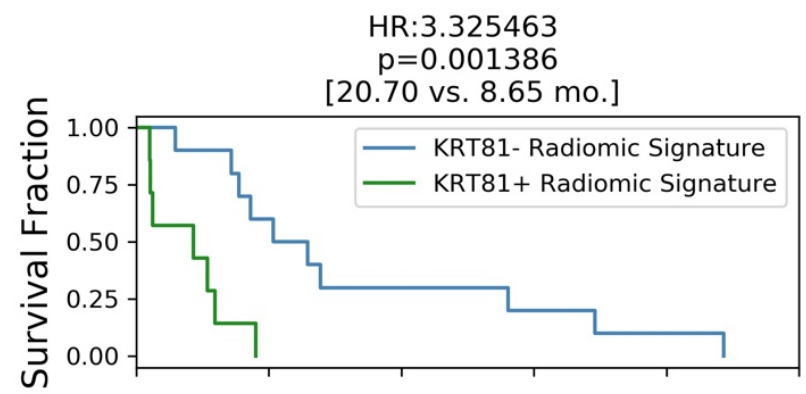

HR: 1.038814

$\mathrm{p}=0.363046$

[32.70 vs. $10.70 \mathrm{mo}$.]

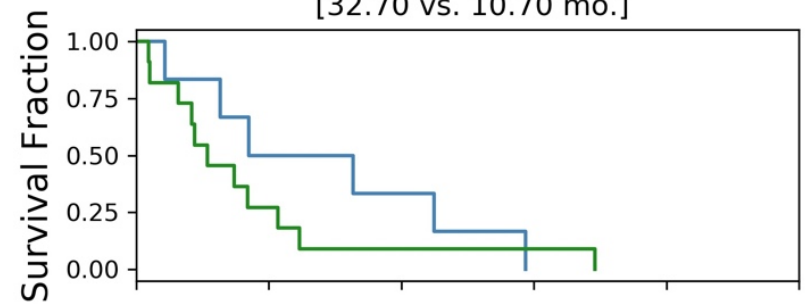

HR: 2.452812

$\mathrm{p}=0.038746$

[12.70 vs. $9.00 \mathrm{mo}$.

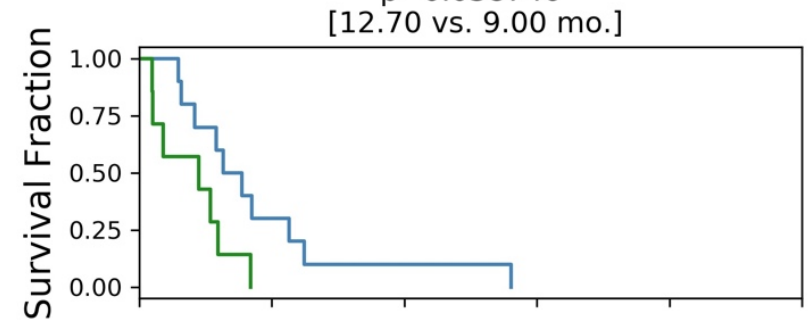

HR:3.177212

$\mathrm{p}=0.004071$

[24.20 vs. $7.90 \mathrm{mo}$.]

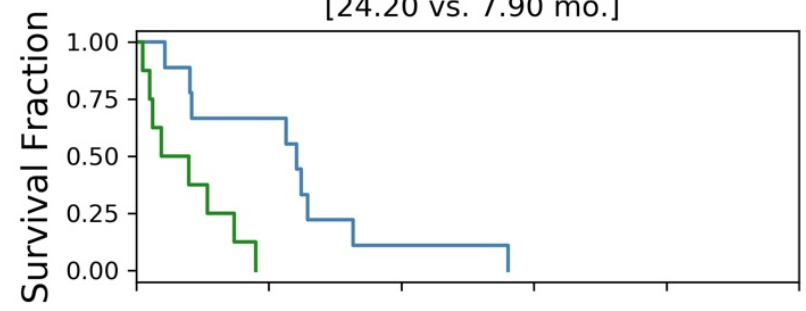

HR:3.148256

$p=0.003430$

[15.50 vs. $3.60 \mathrm{mo}$.]

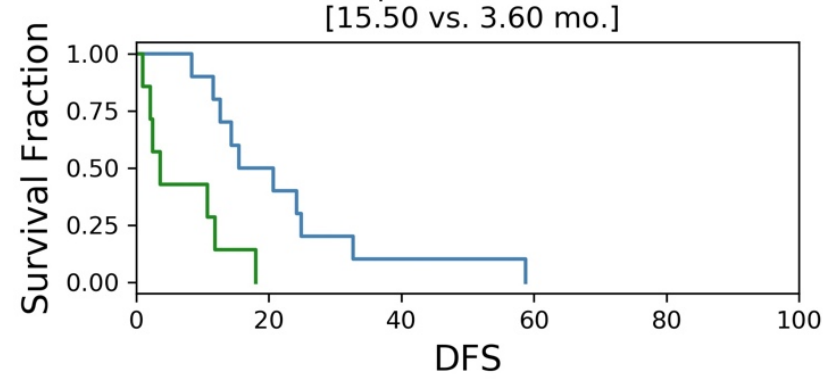

HR:3.025672

$\mathrm{p}=0.005916$

[22.60 vs. 8.79 mo.]

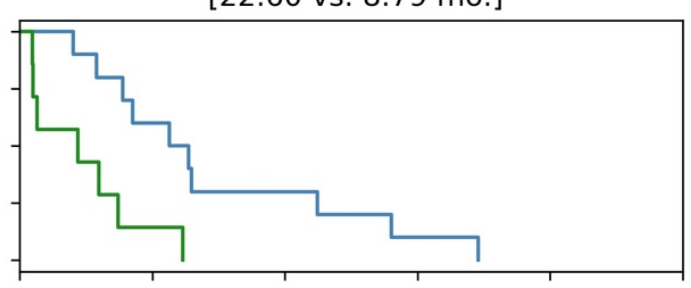

HR:3.379457

$\mathrm{p}=0.001020$

[20.70 vs. $2.20 \mathrm{mo}$.]

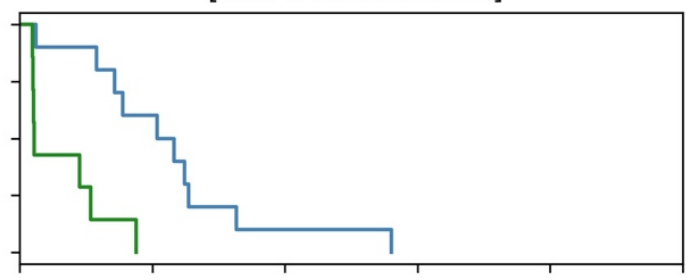

$H R: 3.360382$

$\mathrm{p}=0.001139$

[22.60 vs. $2.44 \mathrm{mo}$.]

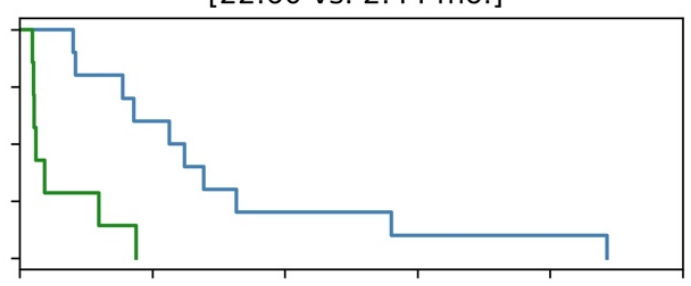

HR: 0.460075

$p=0.485162$

[12.70 vs. $3.60 \mathrm{mo}$.]

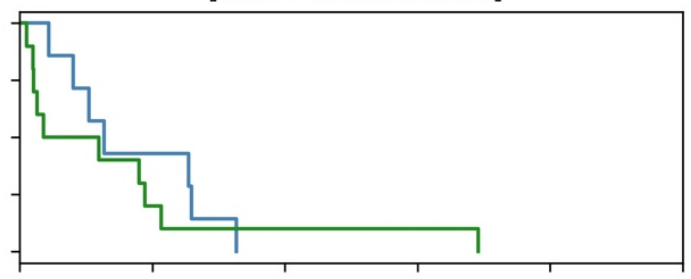

HR:2.635819

$p=0.028424$

[25.50 vs. $10.70 \mathrm{mo}$.]

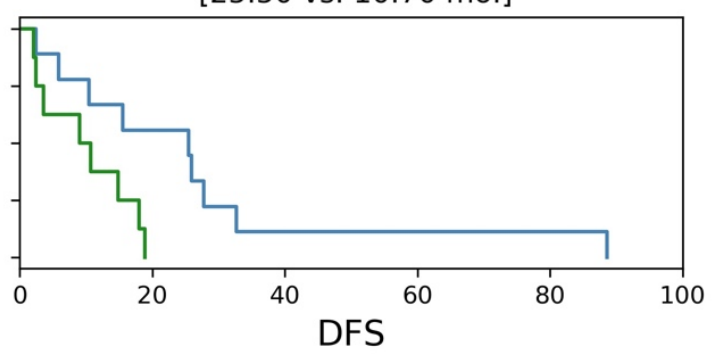

Kaplan-Meier curves resulting from 10-fold cross validation and showing disease-free survival stratified by radiomic signature (KRT81+/high risk (blue line) or KRT81-/low risk (green line)). Total $\mathrm{N}=17$. 


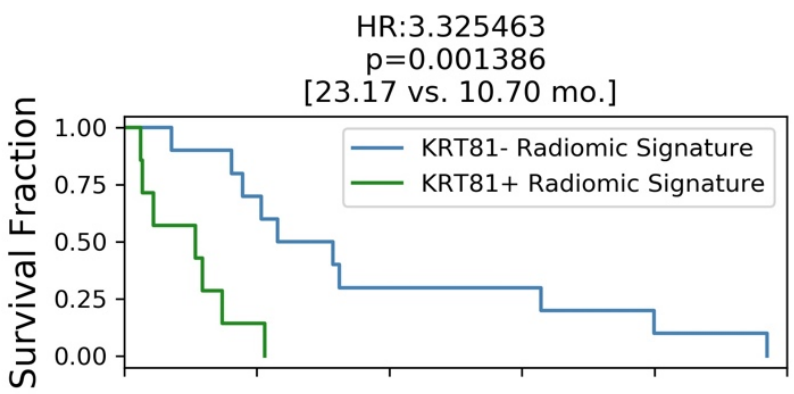

HR: 1.038814

$p=0.363046$

[39.67 vs. $11.77 \mathrm{mo}$.]

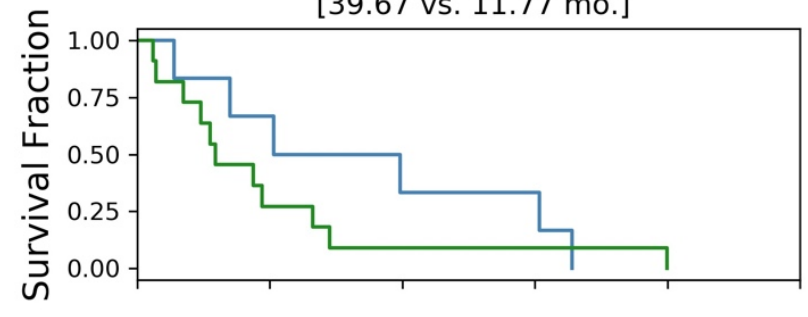

HR: 2.402365

$p=0.043864$

[14.00 vs. $9.30 \mathrm{mo}$.]

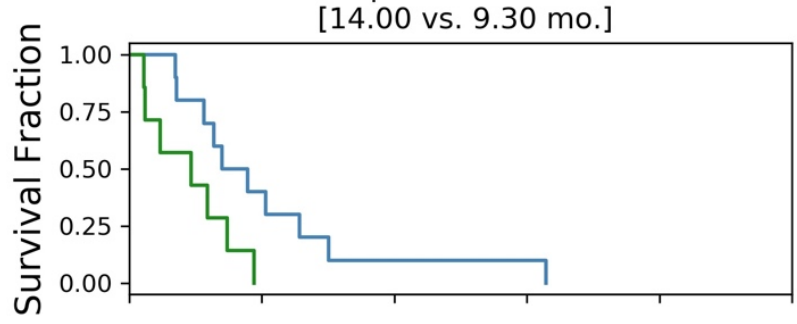

HR:3.075391

$p=0.006333$

[26.57 vs. $9.13 \mathrm{mo}$.

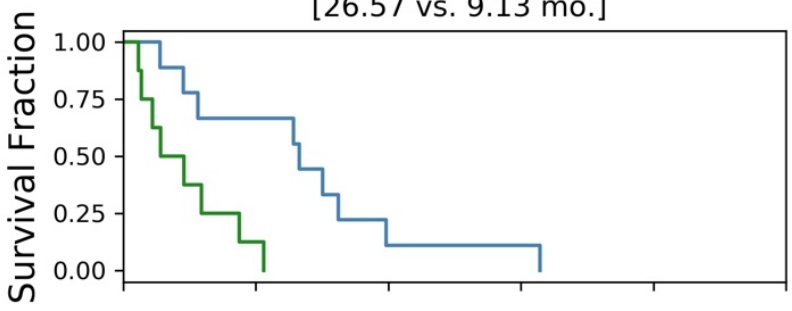

HR:3.058890

$p=0.005134$

[17.83 vs. $4.70 \mathrm{mo}$.]

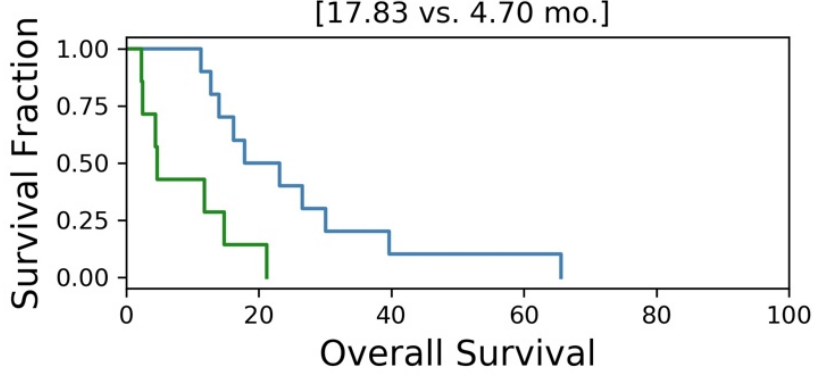

HR:3.025672

$\mathrm{p}=0.005916$

[25.70 vs. $10.97 \mathrm{mo}$.]

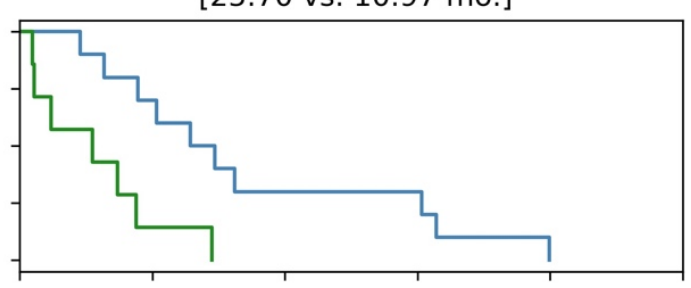

HR:3.269673

$p=0.001874$

[23.17 vs. $2.87 \mathrm{mo}$.

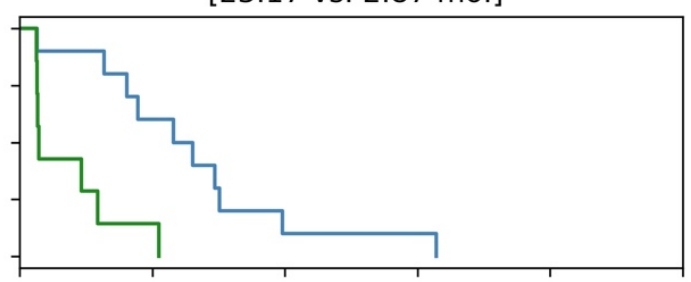

HR:3.360382

$\mathrm{p}=0.001139$

[25.70 vs. $4.37 \mathrm{mo}$.]

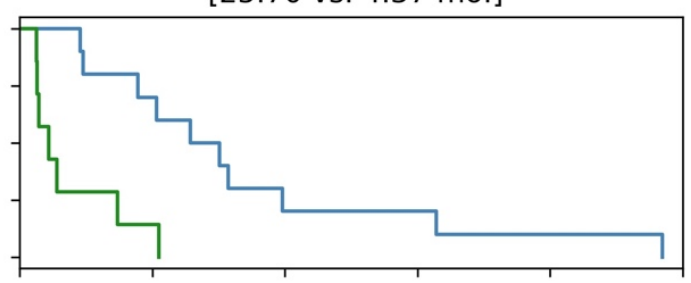

HR:0.273191

$\mathrm{p}=0.524937$

[14.00 vs. $4.77 \mathrm{mo}$.

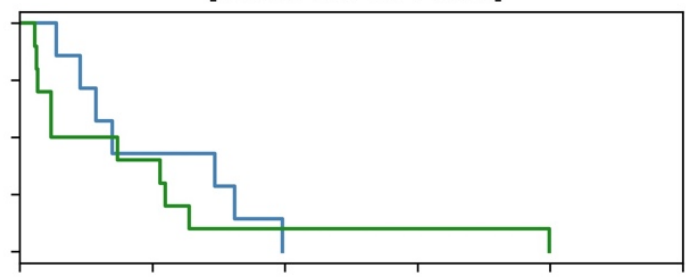

HR:2.635819

$p=0.028424$

[29.47 vs. $11.77 \mathrm{mo}$.]

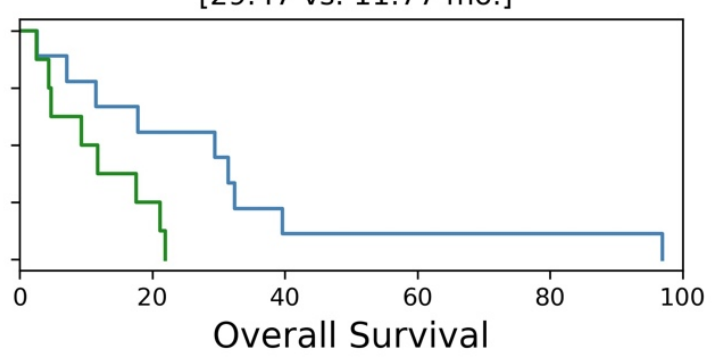

Kaplan-Meier curves resulting from 10-fold cross validation and showing overall survival stratified by radiomic signature. (KRT81+/high risk (blue line) or KRT81-/low risk (green line)). Total $\mathrm{N}=17$. 

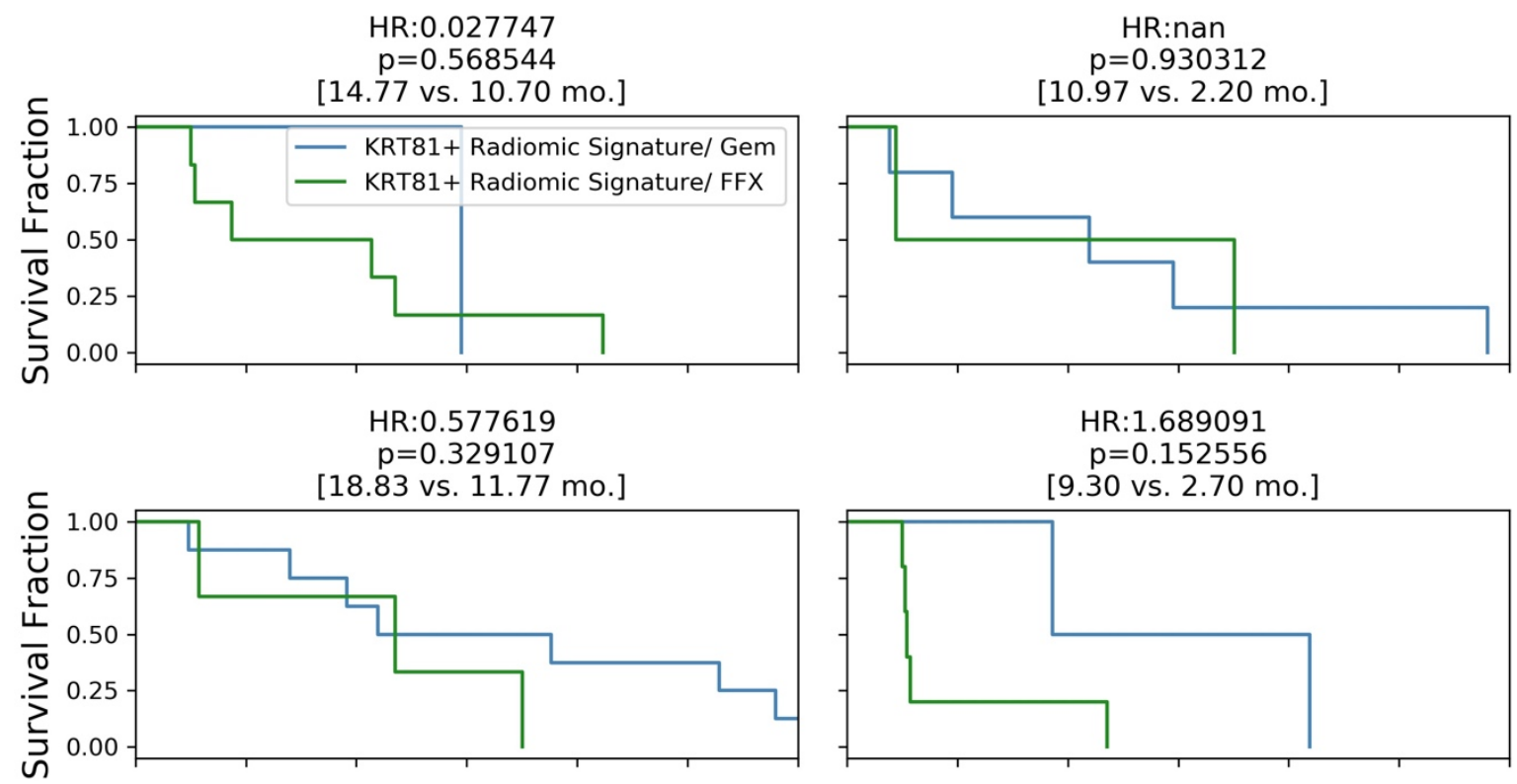

HR: 1.036533

$\mathrm{p}=0.235104$

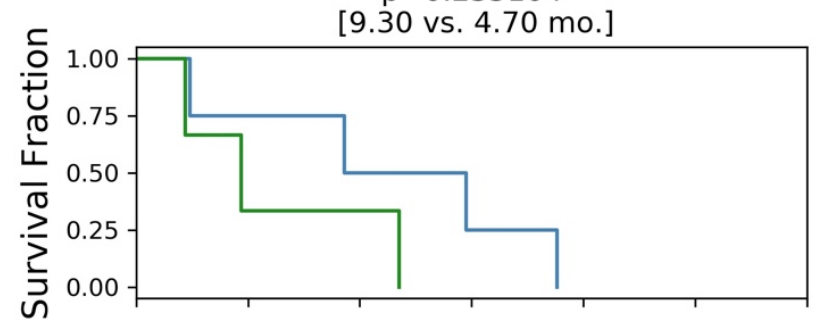

HR:2.564864

$\mathrm{p}=0.017669$

[14.77 vs. $2.63 \mathrm{mo}$.

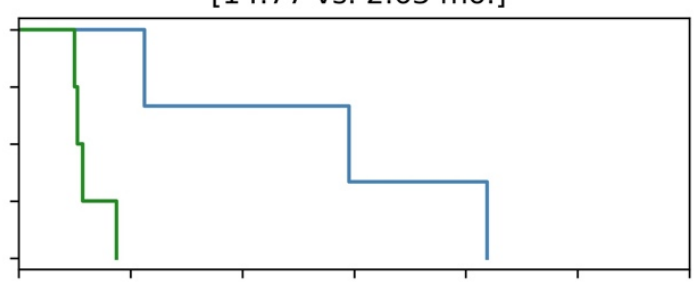

HR:nan

$p=0.637414$

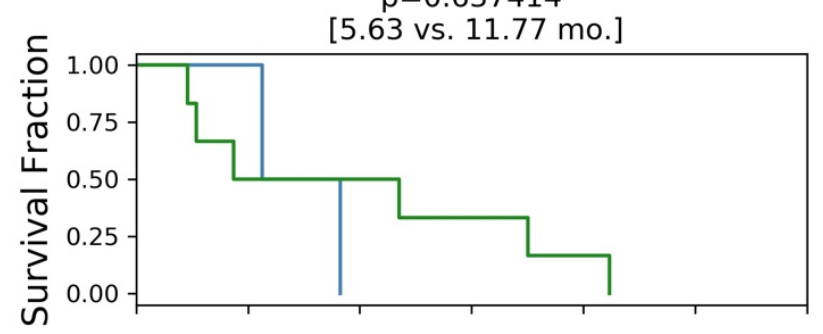

R:2.570622

$\mathrm{p}=0.019809$

[22.00 vs. $2.70 \mathrm{mo}$.

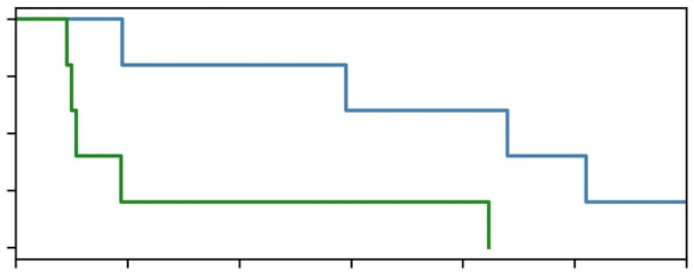

HR:0.027747

$\mathrm{p}=0.568544$

HR: 1.229012

$p=0.271651$

[9.30 vs. $11.77 \mathrm{mo}$.
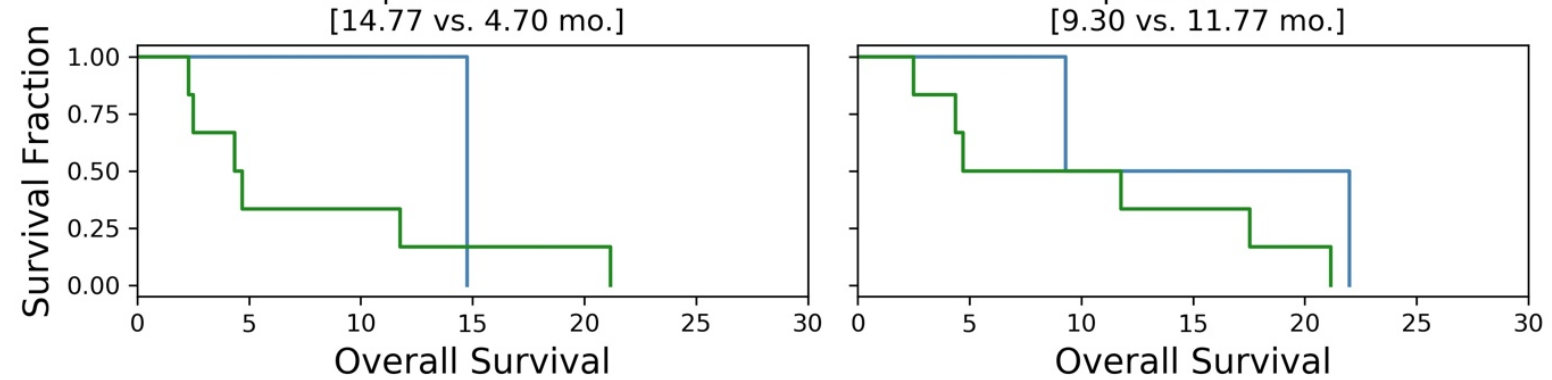

Kaplan-Meier curves resulting from 10 -fold cross validation and showing overall survival stratified by radiomic signature and chemotherapy regimen. (KRT81+/high risk with gemcitabine (blue line) or FOLFIRINOX (green line)). Total N=7 to 11 

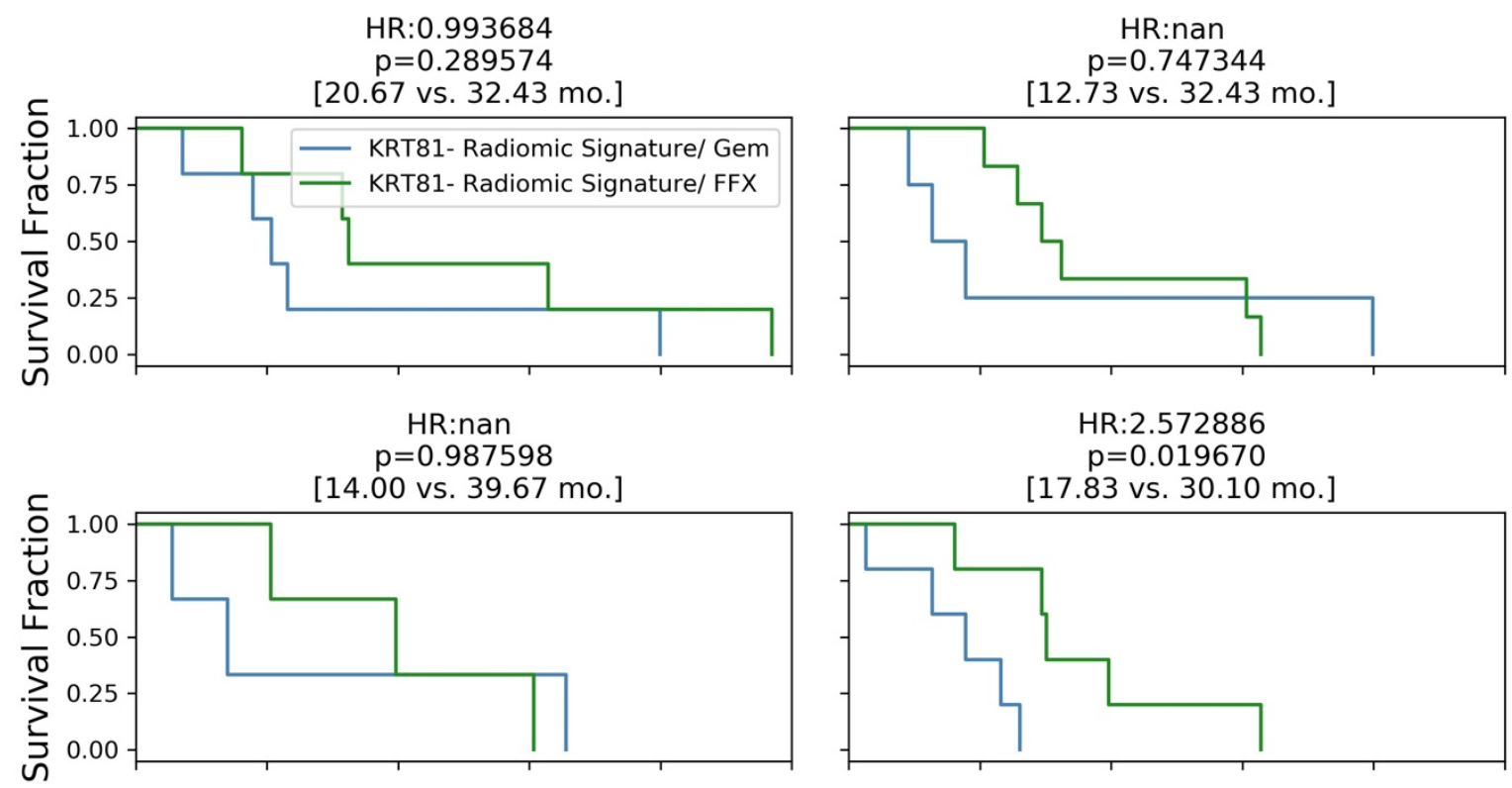

HR:2.897030

$p=0.003777$

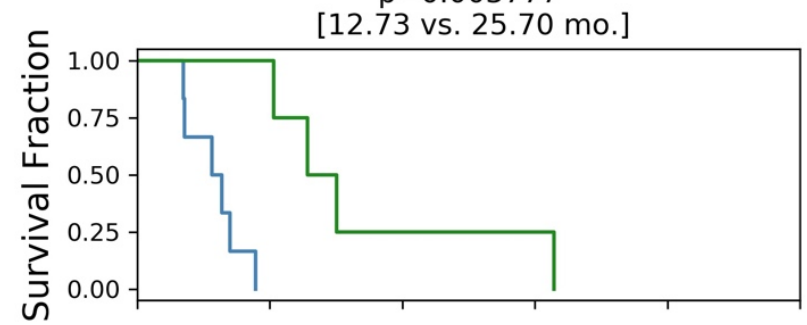

HR:3.342781

$p=0.001074$

[9.57 vs. $39.67 \mathrm{mo}$.

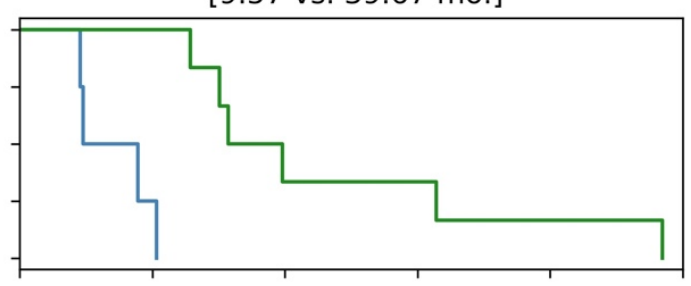

HR:3.269918

$p=0.001827$

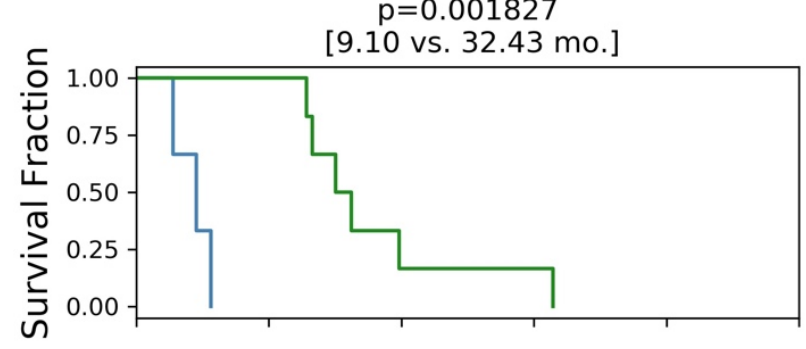

HR:2.421023

$\mathrm{p}=0.017669$

[9.10 vs. $32.43 \mathrm{mo}$.

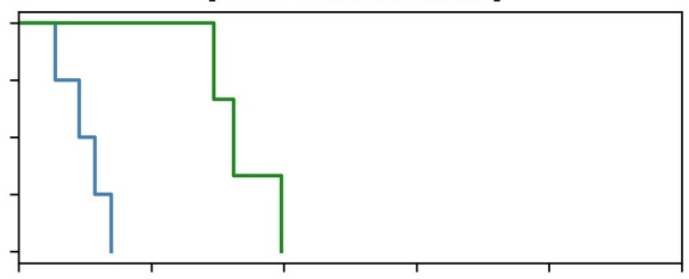

HR:3.096416

$\mathrm{p}=0.002656$

$\mathrm{p}=0.522995$

[17.83 vs. $26.57 \mathrm{mo}$.

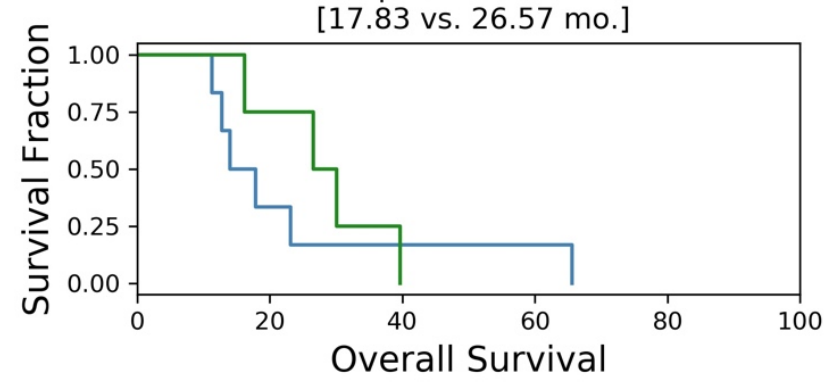

[7.13 vs. $32.43 \mathrm{mo}$.]

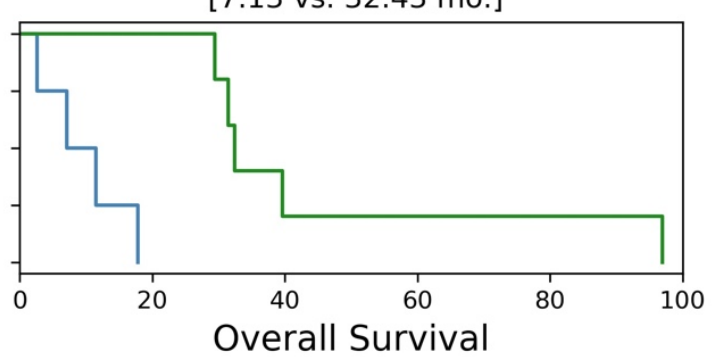

Kaplan-Meier curves resulting from 10 -fold cross validation and showing overall survival stratified by radiomic signature and chemotherapy regimen. (KRT81-/low risk with gemcitabine (blue line) or FOLFIRINOX (green line)). Total N=6 to 10 


\section{STROBE Statement Checklist}

\begin{tabular}{|c|c|c|c|}
\hline & $\begin{array}{c}\text { Item } \\
\text { No }\end{array}$ & Reccomendation & $\begin{array}{l}\text { Remark/ } \\
\text { Location }\end{array}$ \\
\hline \multirow[t]{2}{*}{ Title and abstract } & \multirow[t]{2}{*}{1} & $\begin{array}{l}\text { (a) Indicate the study's design with a commonly } \\
\text { used term in the title or the abstract }\end{array}$ & $\begin{array}{l}\text { Reported in } \\
\text { abstract }\end{array}$ \\
\hline & & $\begin{array}{l}\text { (b) Provide in the abstract an informative and } \\
\text { balanced summary of what was done and what } \\
\text { was found }\end{array}$ & $\begin{array}{l}\text { Reported in } \\
\text { abstract }\end{array}$ \\
\hline \multicolumn{4}{|l|}{ Introduction } \\
\hline Background/rationale & 2 & $\begin{array}{l}\text { Explain the scientific background and rationale for } \\
\text { the investigation being reported }\end{array}$ & Introduction \\
\hline Objectives & 3 & $\begin{array}{l}\text { State specific objectives, including any } \\
\text { prespecified hypotheses }\end{array}$ & Introduction \\
\hline \multicolumn{4}{|l|}{ Methods } \\
\hline Study design & 4 & $\begin{array}{l}\text { Present key elements of study design early in the } \\
\text { paper }\end{array}$ & $\begin{array}{l}\text { Mehods/ Study } \\
\text { Design }\end{array}$ \\
\hline Setting & 5 & $\begin{array}{l}\text { Describe the setting, locations, and relevant dates, } \\
\text { including periods of recruitment, exposure, follow- } \\
\text { up, and data collection }\end{array}$ & Ibid. \\
\hline \multirow[t]{2}{*}{ Participants } & \multirow[t]{2}{*}{6} & $\begin{array}{l}\text { (a) Give the eligibility criteria, and the sources and } \\
\text { methods of selection of participants. Describe } \\
\text { methods of follow-up }\end{array}$ & Ibid. \\
\hline & & $\begin{array}{l}\text { (b) For matched studies, give matching criteria and } \\
\text { number of exposed and unexposed }\end{array}$ & Ibid. \\
\hline Variables & 7 & $\begin{array}{l}\text { Clearly define all outcomes, exposures, predictors, } \\
\text { potential confounders, and effect modifiers. Give } \\
\text { diagnostic criteria, if applicable }\end{array}$ & $\begin{array}{l}\text { Ibid. and } \\
\text { Methods/ } \\
\text { Clinical } \\
\text { variables. } \\
\text { Compare also } \\
\text { Results }\end{array}$ \\
\hline $\begin{array}{l}\text { Data sources/ } \\
\text { measurement }\end{array}$ & $8^{*}$ & $\begin{array}{l}\text { For each variable of interest, give sources of data } \\
\text { and details of methods of assessment } \\
\text { (measurement). Describe comparability of } \\
\text { assessment methods if there is more than one } \\
\text { group }\end{array}$ & $\begin{array}{l}\text { Methods/ } \\
\text { Study Design }\end{array}$ \\
\hline Bias & 9 & $\begin{array}{l}\text { Describe any efforts to address potential sources } \\
\text { of bias }\end{array}$ & Results \\
\hline Study size & 10 & Explain how the study size was arrived at & $\begin{array}{l}\text { Methods/ } \\
\text { Study Design }\end{array}$ \\
\hline $\begin{array}{l}\text { Quantitative } \\
\text { variables }\end{array}$ & 11 & $\begin{array}{l}\text { Explain how quantitative variables were handled in } \\
\text { the analyses. If applicable, describe which } \\
\text { groupings were chosen and why }\end{array}$ & $\begin{array}{l}\text { Results, Table } \\
1\end{array}$ \\
\hline \multirow[t]{5}{*}{ Statistical methods } & \multirow[t]{5}{*}{12} & $\begin{array}{l}\text { (a) Describe all statistical methods, including those } \\
\text { used to control for confounding }\end{array}$ & Methods \\
\hline & & $\begin{array}{l}\text { (b) Describe any methods used to examine } \\
\text { subgroups and interactions }\end{array}$ & Ibid. \\
\hline & & (c) Explain how missing data were addressed & $\begin{array}{l}\text { Supplementary } \\
\text { Material } \\
\text { Results }\end{array}$ \\
\hline & & $\begin{array}{l}\text { (d) If applicable, explain how loss to follow-up was } \\
\text { addressed }\end{array}$ & N.A. \\
\hline & & (e्) Describe any sensitivity analyses & $\begin{array}{l}\text { Supplementary } \\
\text { Material }\end{array}$ \\
\hline \multicolumn{3}{|l|}{ Results } & \\
\hline
\end{tabular}




\begin{tabular}{|c|c|c|c|}
\hline \multirow[t]{3}{*}{ Participants } & \multirow[t]{3}{*}{$13^{*}$} & $\begin{array}{l}\text { (a) Report numbers of individuals at each stage of } \\
\text { study-eg numbers potentially eligible, examined } \\
\text { for eligibility, confirmed eligible, included in the } \\
\text { study, completing follow-up, and analysed }\end{array}$ & $\begin{array}{l}\text { Methods/ } \\
\text { Study Design } \\
\text { Supplementary } \\
\text { Material }\end{array}$ \\
\hline & & $\begin{array}{l}\text { (b) Give reasons for non-participation at each } \\
\text { stage }\end{array}$ & Ibid. \\
\hline & & (c) Consider use of a flow diagram & See below \\
\hline \multirow[t]{3}{*}{ Descriptive data } & \multirow[t]{3}{*}{$14^{*}$} & $\begin{array}{l}\text { (a) Give characteristics of study participants (eg } \\
\text { demographic, clinical, social) and information on } \\
\text { exposures and potential confounders }\end{array}$ & $\begin{array}{l}\text { Methods/ } \\
\text { Study design } \\
\text { Results, Table } \\
2\end{array}$ \\
\hline & & $\begin{array}{l}\text { (b) Indicate number of participants with missing } \\
\text { data for each variable of interest }\end{array}$ & N.A. \\
\hline & & $\begin{array}{l}\text { (c) Summarise follow-up time (eg, average and } \\
\text { total amount) }\end{array}$ & $\begin{array}{l}\text { Methods/ } \\
\text { Study design }\end{array}$ \\
\hline Outcome data & $15^{*}$ & $\begin{array}{l}\text { Report numbers of outcome events or summary } \\
\text { measures over time }\end{array}$ & Results \\
\hline \multirow[t]{3}{*}{ Main results } & \multirow[t]{3}{*}{16} & $\begin{array}{l}\text { (a) Give unadjusted estimates and, if applicable, } \\
\text { confounder-adjusted estimates and their precision } \\
\text { (eg, 95\% confidence interval). Make clear which } \\
\text { confounders were adjusted for and why they were } \\
\text { included }\end{array}$ & Results \\
\hline & & $\begin{array}{l}\text { (b) Report category boundaries when continuous } \\
\text { variables were categorized }\end{array}$ & N.A. \\
\hline & & $\begin{array}{l}\text { (c) If relevant, consider translating estimates of } \\
\text { relative risk into absolute risk for a meaningful time } \\
\text { period }\end{array}$ & N.A. \\
\hline Other analyses & 17 & $\begin{array}{l}\text { Report other analyses done-eg analyses of } \\
\text { subgroups and interactions, and sensitivity } \\
\text { analyses }\end{array}$ & Results \\
\hline \multicolumn{4}{|l|}{ Discussion } \\
\hline Key results & 18 & $\begin{array}{l}\text { Summarise key results with reference to study } \\
\text { objectives }\end{array}$ & Discussion \\
\hline Limitations & 19 & $\begin{array}{l}\text { Discuss limitations of the study, taking into account } \\
\text { sources of potential bias or imprecision. Discuss } \\
\text { both direction and magnitude of any potential bias }\end{array}$ & Discussion \\
\hline Interpretation & 20 & $\begin{array}{l}\text { Give a cautious overall interpretation of results } \\
\text { considering objectives, limitations, multiplicity of } \\
\text { analyses, results from similar studies, and other } \\
\text { relevant evidence }\end{array}$ & Discussion \\
\hline Generalisability & 21 & $\begin{array}{l}\text { Discuss the generalisability (external validity) of the } \\
\text { study results }\end{array}$ & $\begin{array}{l}\text { Introduction } \\
\text { Discussion } \\
\text { Supplementary } \\
\text { Material } \\
\end{array}$ \\
\hline \multicolumn{4}{|c|}{ Other information } \\
\hline Funding & 22 & $\begin{array}{l}\text { Give the source of funding and the role of the } \\
\text { funders for the present study and, if applicable, for } \\
\text { the original study on which the present article is } \\
\text { based }\end{array}$ & Preamble \\
\hline
\end{tabular}


The flowchart of patient recruitment is shown below:

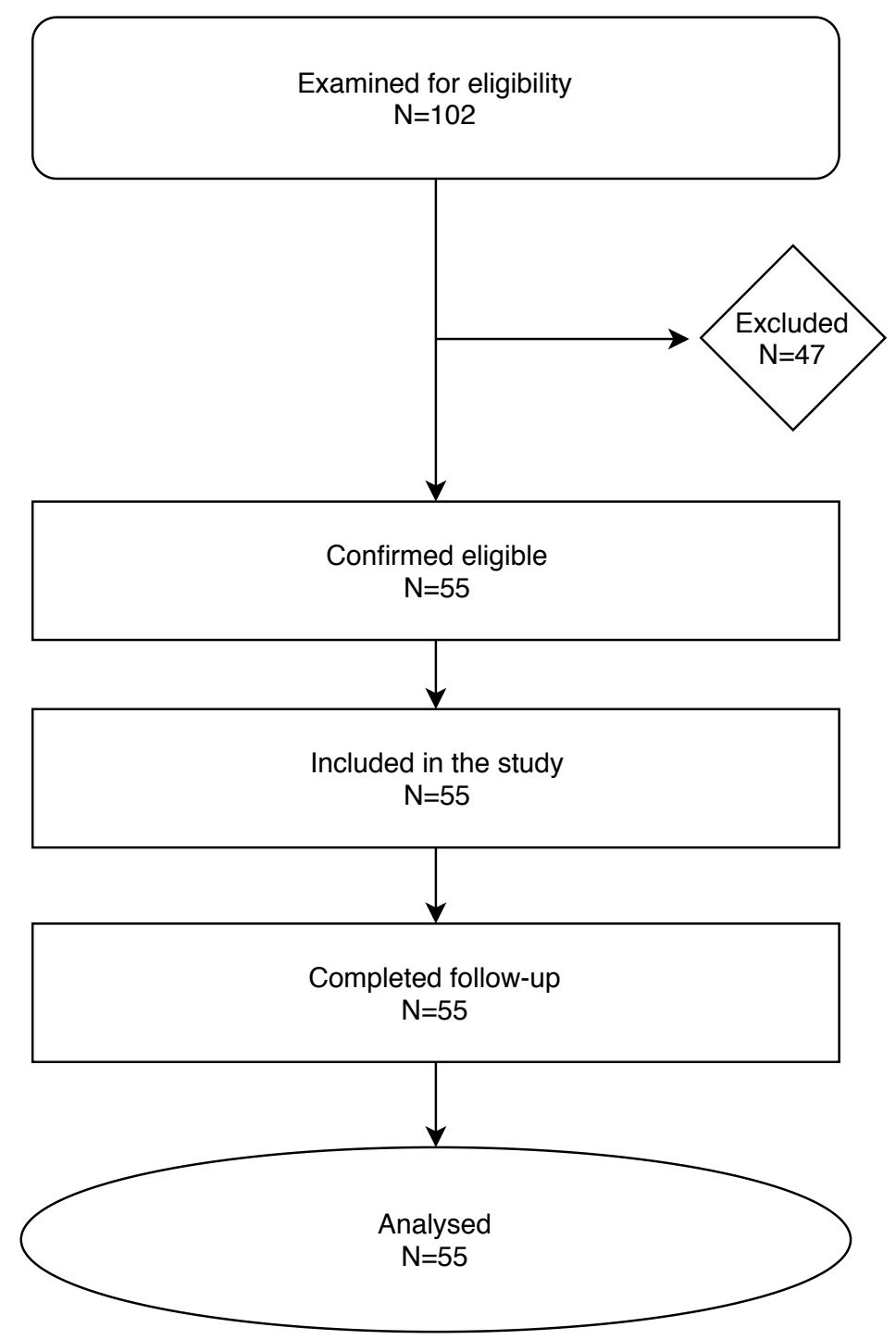

Reasons for exclusion:

No PDAC in final histopathological report (8 patients), insufficient or incomplete imaging (14 patients), death within 2 months of imaging (4 patients), received prior treatment (8 patients), histopathologically unclassifiable tumor subtype (13 patients) 


\section{Supplementary Bibliography}

(1) Vallieres, C. R. Freeman, S. R. Skamene, and I. El Naqa. A radiomics model from joint FDG-PET and MRI texture features for the prediction of lung metastases in softtissue sarcomas of the extremities.Physics in medicine and biology, 60(14):5471-96, Jul 2015

(2) van Griethuysen, J. J. M., Fedorov, A., Parmar, C., Hosny, A., Aucoin, N., Narayan, V., Beets-Tan, R. G. H., Fillon-Robin, J. C., Pieper, S., Aerts, H. J. W. L. (2017). Computational Radiomics System to Decode the Radiographic Phenotype. Cancer Research, 77(21), e104-e107

Compare also: https://pyradiomics.readthedocs.io/

(3) PyRadiomics feature definitions:

https://pyradiomics.readthedocs.io/en/latest/features.html

Compare also: (4) below

(4) Zwanenburg, A., Leger, S., Vallières, M., and Löck, S. (2016). Image biomarker standardisation initiative: $\underline{\text { https://arxiv.org/abs/1612.07003v7 }}$

Multiple versions of the manual are available, version 7 is linked and was current at the time of the analysis

(5) McGraw, K. O., \& Wong, S. P. (1996). Forming inferences about some intraclass correlation coefficients. Psychological Methods, 1(1), 30-46.

(6) Dancey C.P., Reidy J. Statistics for Psychology, Pearson Education; 2007 